\title{
Classification, Identification, and Analysis of Events on Twitter Through Data Mining
}

\author{
Autores \\ Jesús Silva, Pedro Berdejo, Yuki Higa, Juan Manuel Cera Visbal, Danelys Cabrera, Alexa Senior \\ Naveda, Yasmin Flores, Omar Bonerge Pineda Lezama
}

\begin{abstract}
Due to its popularity, Twitter is currently one of the major players in the global network, which has established a new form of communication: the microblogging. Twitter has become an essential media network for the follow-up, diffusion and coordination of events of diverse nature and importance (Gonzalez-Agirre et al. in Multilingual central repository version 3.0. Proceedings of the Eight International Conference on Language Resources and Evaluation (LREC'12). Istanbul, Turkey, 2012, [1]), such as a presidential campaign, a disaster situation, a war or the repercussion of information. In such scenario, it is considered a relevant source of information to know the opinions that are emitted about different issues or people. This research proposes the evaluation of several supervised classification algorithms to address the problem of opinion mining on Twitter.
\end{abstract}

Palabras clave

Machine learning, Twitter, Opinion mining, Classification 